Chimia 45 (1991) 285-287

() Schweiz. Chemiker-Verband; ISSN 0009-4293

\title{
Application of Dyes in Nonlinear Optical Materials
}

\author{
Michael G. Hutchings*, Simon Allen, D. John Bone, Andrew N. Burgess, \\ Neil Carter, David P. Devonald, Archie Eaglesham, E. Sarah Froggat, \\ Timothy G. Ryan, Robert B. Sampson, and Ronald M. Swart
}

Abstract. An outline of some azobenzene derivatives which are being used in ICI as the basis for new nonlinear optical materials is presented.

\section{Introduction}

The advent of high-powered lasers has given rise to new optics-based information processing technologies of huge potential commercial value. These revolve around data storage, data transfer, and data processing. From the chemist's point of view, these are characterised by the active materials which are necessary to interact with the electromagnetic radiation, or 'light', which is the data-carrying medium. Optical data transfer (optical communications) depends on materials which have nonlinear optical (NLO) properties [1]. This paper gives a brief overview of their general characteristics, especially from a dyestuff point of view, and will outline some of those recently investigated in the ICI laboratories.

\section{Nonlinear Optical Materials and Dye-} stuffs

NLO materials have several physically observable properties which are not present in non-NLO species. For example, second order NLO materials change their refractive index in an electric field (electro-optic modulator applications), and can cause frequency doubling of impinging light (new lasers; also used for characterisation). The background to materials with NLO activity has appeared in many publications (e.g. [1]). The second order NLO activity of a molecule is characterised by the first hyperpolarisability $\beta$ : higher values of $\beta$ imply more efficient NLO activity. The central question is how $\beta$ varies for different molecular structures.

The most important molecular characteristic is that only molecules which are dipolar have a nonzero value of $\beta$. In other words, centrosymmetric molecules have no NLO activity.

The arrangement of the molecules on the macroscopic level is also crucial to NLO activity. The molecules themselves must be organised such that the ensemble must have nocentre of symmetry. Thus, if the molecules are arranged randomly, or in such a way that their dipole moment vectors are pairwise antiparallel, then the material will again have no NLO activity. It is, therefore, necessary to ensure that there is a degree of order of the molecular dipoles.

The requirements for both molecular and macroscopic acentricity characterise the design and fabrication of materials with NLO activity.
It has been found that the magnitude of $\beta$ is increased if the dipolarity of the molecule is associated with a $\pi$-delocalised system, containing a $\pi$-donor conjugated with a $\pi$ acceptor. Thus, the archetypal NLO molecule could be regarded as $p$-nitroaniline. Furthermore, the NLO activity is increased for stronger $\pi$-donors and-acceptors, and in general increases with the conjugation path length between the donor and acceptor. Example values of $\beta$ for a range of molecules are shown in Fig. 1 .

It is immediately apparent that this generic structure type is that found in many dyestuff classes, such as donor-acceptor substituted azobenzenes. Such molecules would be expected to show NLO activity, as long as the individual molecules were arranged acentrically. On the other hand, there are other dyestuffs which have a centre of molecular symmetry, such as indigo or cyanines, and which, therefore, are not normally NLO active.

The main property of dyestuffs - their absorbance of visible light to give colour - is not of direct relevance to the question of NLO properties. However, the performance of an NLO active molecule in a pirticular application can be dependent on the colour. The effect can be beneficial or deleterious, depending on the circumstances.

Interest in organic molecules for NLO study has increased for several reasons, including the very wide range of structural modifications which are possible, the ease with which these can be synthesised, and the ease and cheapness of fabrication into active materials, leading to convenient device fabrication. There are further advantages in

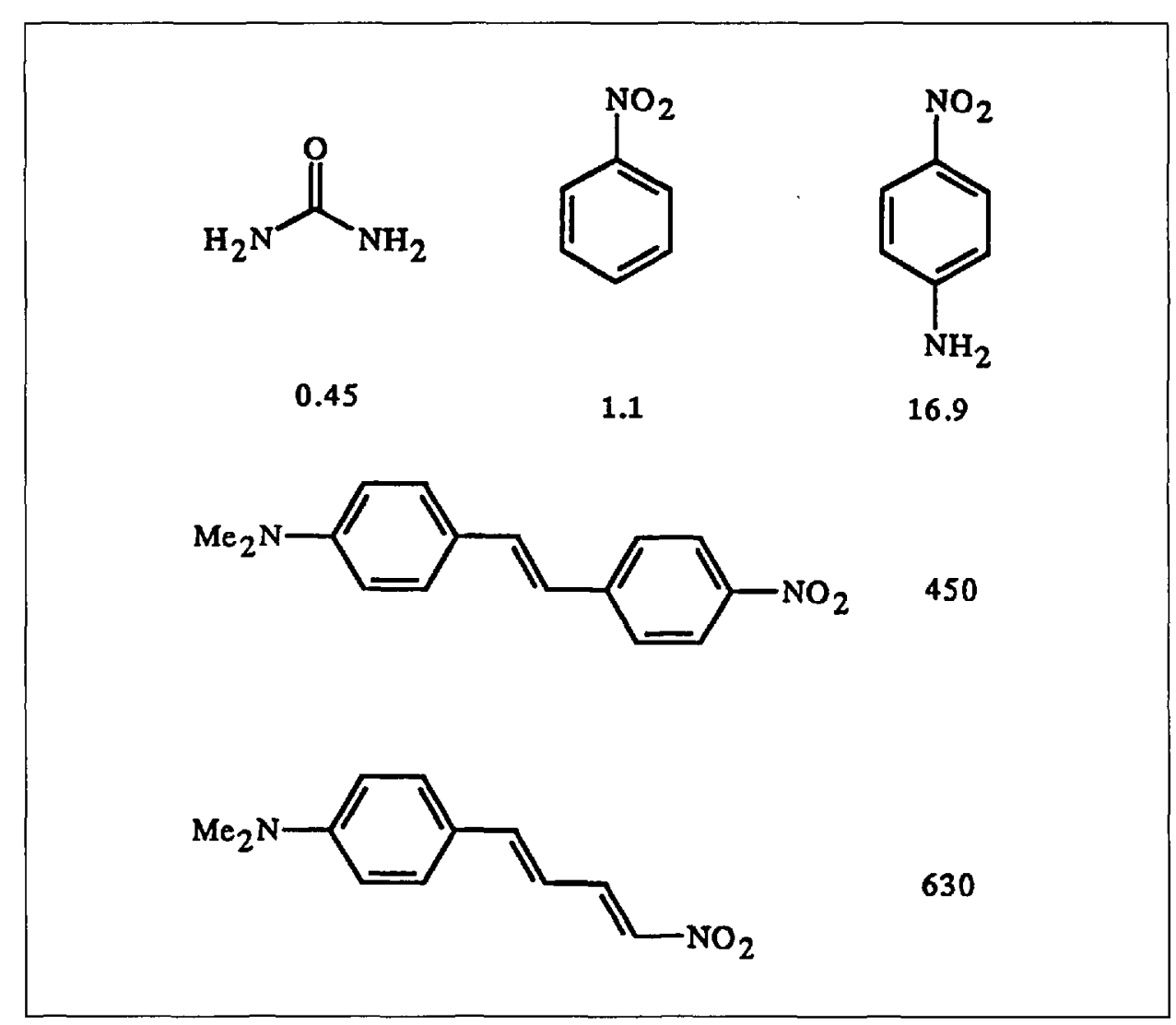


basing NLO materials on known dyestuff chromophores, particularly azobenzenes. They are easy to synthesise with the required types of substituents, and easy to functionalise further. They normally have good chemical stability, and so withstand further reactions such as polymerisation, and yet can be bleached under appropriate conditions.

The fabrication of NLO active materials raises the all-important question of how the necessary order can be imposed on the constituent NLO active molecules. For convenience, three methods will be considered, with examples from ICI work:

- crystals

- poled polymer films

- Langmuir-Blodgett films.

\section{Crystals as NLO Materials}

The most obvious means of enforcing order on an ensemble of molecules is to crystallise them. Crystal packing can have two results. Either the constituent molecules form into a centrosymmetric array, where the molecular dipole vectors are arranged head-to-head and tail-to-tail. Alternatively, the molecules can pack into a noncentrosymmetric arrangement. In the former case, the NLO activity will be zero. Unfortunately, this is by far the commonest mode of packing for dipolar organic molecules. The latter arrangement can lead to NLO activity, although the magnitude will be dependent on the molecular species, and their exact arrangement in the crystal.

Many crystals have been studied for NLO activity. However, the major drawback is that currently it is impossible to predict how a particular molecule will pack in the crystalline phase. Moreover, it is impossible, or at least very difficult, to extrapolate from known crystal structures how a modification to the molecular structure will affect the crystal packing. For example $p$-nitroaniline

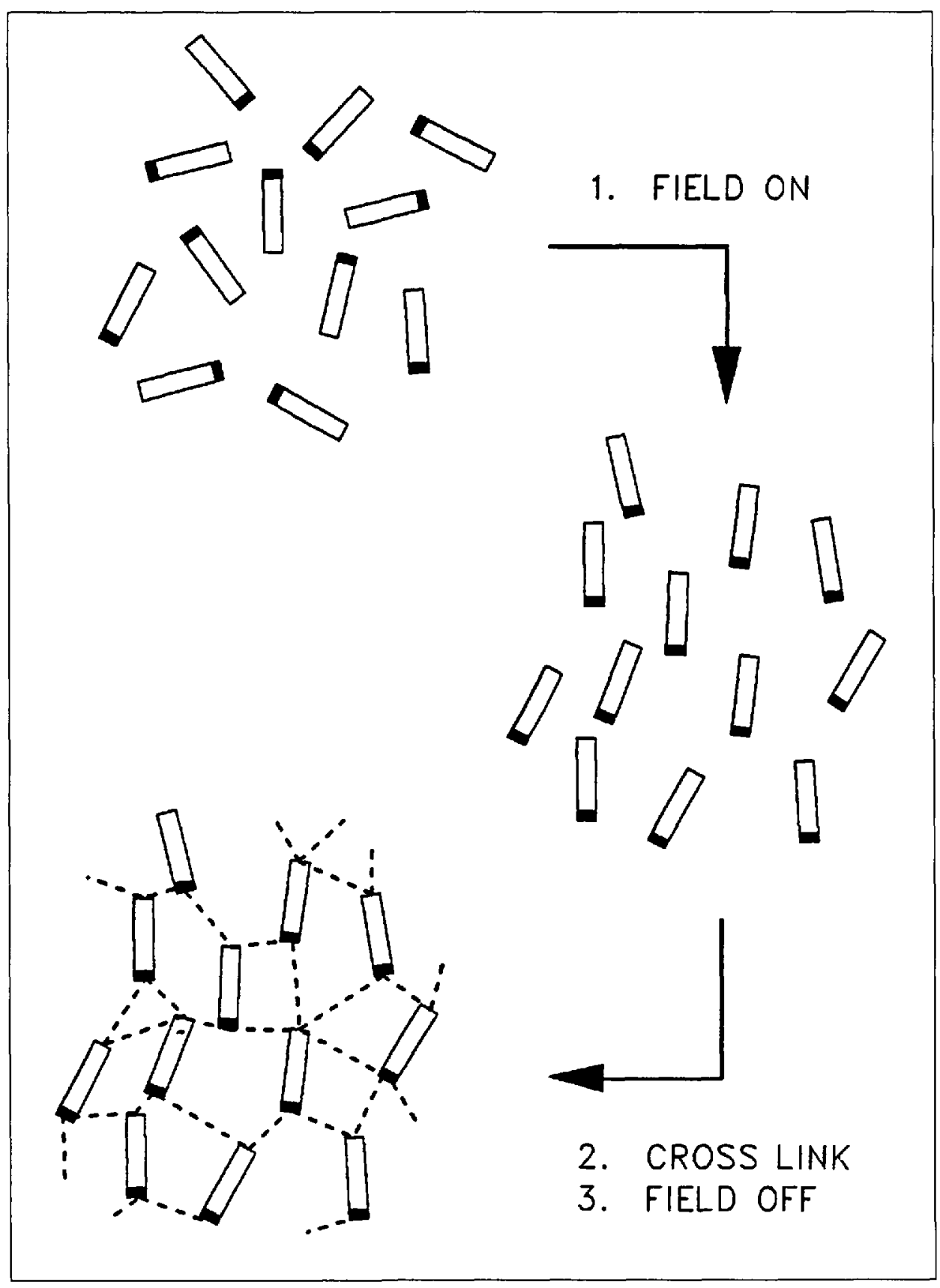

Fig. 2. Preparation of stable-ardered NLO films by poling of NLO chromophores and cross-linking

gives a centrosymmetric, and thus NLO inactive, crystal. However, the very closely related 2-methyl-4-nitroaniline packs noncentrosymmetrically, with the dipole vectors of all molecules more or less pointing in the same direction. Crystals of this molecule are consequently NLO active. Crystal engineering, where structural modification is intended to affect packing, is being actively developed, but as yet without general success.

In ICI, appropriate crystalline samples are routinely screened for NLO activity, by measuring their ability to cause second harmonic generation. From 200 random samples recently measured, only 25 showed any NLO activity at all. Of the 200,74 were conventional dyes. Only two of these were NLO active, indicating the high preference such molecules have for forming centrosymmetric arrays in the crystal.

\section{Poled Polymer Films as NLO Materials}

Dipolar molecules, spread as a thin film on an inactive substrate, will adopt a random orientation, and thus be NLO inactive. However, if they are placed in a strong electric field, they will tend to align. As a result, the film will demonstrate NLO activity. The requirement is for the material to retain its NLO activity more or less permanently in the absence of the poling field, even when exposed to periods at elevated temperatures. It is, therefore, necessary to lock in the order caused by poling. The approach currently being investigated in ICI is to cross-link functionalised donor-acceptor substituted azobenzenes in the presence of a strong poling field $(\mathrm{ca} .10 \mathrm{kV}$ corona poling potential across a $2-\mu \mathrm{m}$ thick film) (Fig. 2).

It has been found that both di- and tetrafunctionalised dyes, such as $\mathbf{1}$ and $\mathbf{2}$ shown in Fig. 3 can be taken through this process to give stable NLO active films. The activity of each is retained essentially indefinitely at room temperature. However, because of the extra degree of cross-linking due to the increased number of methacrylate units, the films given by azo dye 2 retain their NLO activity at higher temperatures (Fig. 3). The chemical, mechanical, optical, and NLO properties of films based on $\mathbf{2}$ are attractive for application in electro-optic modulators. Related species based on different chromophores and different cross-linking mechanisms are being further investigated.

\section{Langmuir-Blodgett Films as NLO Materials}

The Langmuir-Blodgett (LB) technique involves transfer of ordered monolayers from water, to a solid substrate, such as glass. By repetition of the technique, multilayers of molecules can be built up, in a very regular way. Molecules which form LB films are amphiphilic: characterised by the presence of a polar head group at one end of the 
molecule, and a hydrophobic unit at the other, ensuring the required alignment in the monolayer. Subsequent layers can build up in two ways: either head-to-tail (Z-type), or head-to-head (Y-type). The latter is usually thermodynamically more stable, and, therefore, the more common. If an NLO chromophore is incorporated into the amphiphile, the order imposed by the LB technique ensures that the monolayer, and also Z-type multilayers, are NLO active. On the other hand a Y-type structure is not NLO active, since alternate layers of molecular dipoles point in opposite directions.

In order to address some of the problems associated with LB film stability, and the tendency for defects to form in the multilayer structures, polymers have been used as materials for LB films. In ICI, recent work has concentrated on oligomeric species, part way between conventional monomeric chromophores and polymers. The novel diamide-linked trisazobenzene 3 is typical. This forms excellent quality LB films, which are mechanically and chemically stable. The NLO activity of a monolayer is high, but unfortunately 3 gives Y-type multilayer structures. To overcome this problem, a corresponding molecule with inverted dipole, to be alternated with layers of 3 , is being studied.

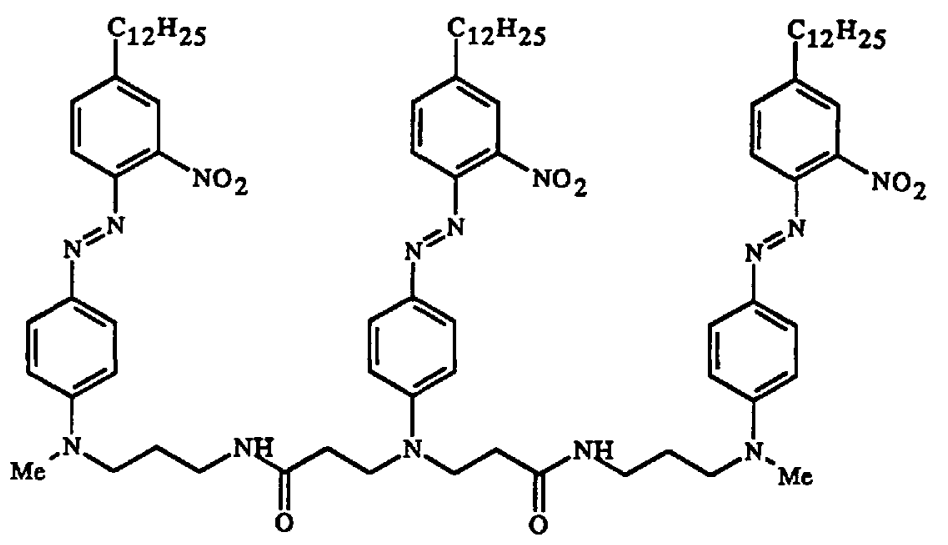

A detailed picture of the alignment and conformation of $\mathbf{3}$ and related molecules in the LB phase has been built up, largely based on neutron scattering and molecular modelling studies.

3

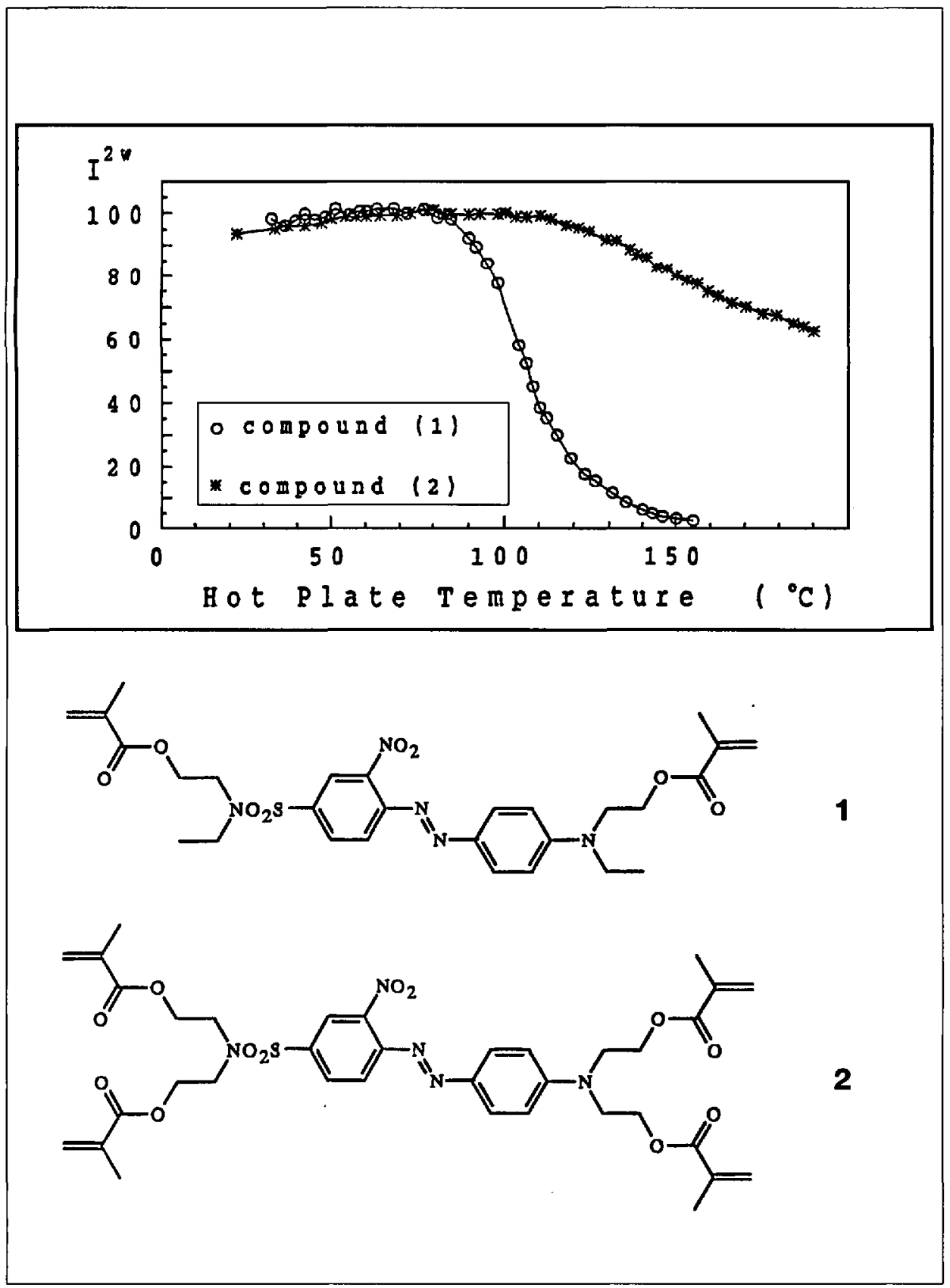

\section{Conclusion}

This short paper only summarises a few aspects of the NLO properties of molecules currently under study in ICI. Furthermore, materials which have third order NLO activity have been ignored. More detailed discussions of NLO materials and the phenomenon in general can be found in [1] and [2]. Aspects of this work are the subject of ICI patent applications.

[1] P.N. Prasad, D.J. Williams, 'Introduction to Nonlinear Optical Effects in Molecules and Polymers', J. Wiley and Sons, Chichester, 1991.

[2] 'Materials for Nonlinear Optics. Chemical Perspectives', Eds. S.R. Marder, J.E. Sohn, and G.D. Stucky, ACS, Washington, D.C., 1991. 